\section{Multi-Frame Blind Deconvolution of Atmospheric Turbulence Degraded Images with Mixed Noise Models}

\author{
Afeng Yang, Xue Jiang and David Day-Uei Li
}

This letter proposes a mixed noise model and uses the multi-frame blind deconvolution to restore the images of space objects under the Bayesian inference framework. To minimize the cost function, an algorithm based on iterative recursion was proposed. In addition, three limited bandwidth constraints of the point spread functions were imposed into the solution process to avoid converging to local minima. Experimental results show that the proposed algorithm can effectively restore the turbulence degraded images and alleviate the distortion caused by the noise.

Introduction: Space object surveillance plays a fundamental and critical role in future space exploration. Images of space objects are usually acquired with ground-based telescopes. The image resolution, however, is limited due to the presence of the atmospheric turbulence (which causes the uneven distribution of the refractive index and leads to the wavefront distortion). This greatly deteriorates the quality and resolution of the images. A powerful approach called multi-frame blind deconvolution (MFBD) can significantly reduce the impact of atmospheric turbulence on an imaging system. MFBD can simultaneously estimate the unblurred object and the point spread functions (PSF) from a set of observed noise-inflicted images. The key step of applying MFBD is to accurately introduce a priori information in the restoration process. Many MFBD algorithms and theoretical results have been developed; they used different a priori information in image restoration. Conventional MFBD algorithms usually assume that the observed images are corrupted by a single type of noise, either Poisson noise [1-3] or Gaussian noise [4, 5]. Instead of adopting these strategies, we propose a novel multi-frame image restoration algorithm by adopting a mixed noise model (MFRAM); MFRAM can achieve a faster convergence, reduce noise more effectively and preserve more image details. The flowchart of MFRAM is shown in Fig. 1.

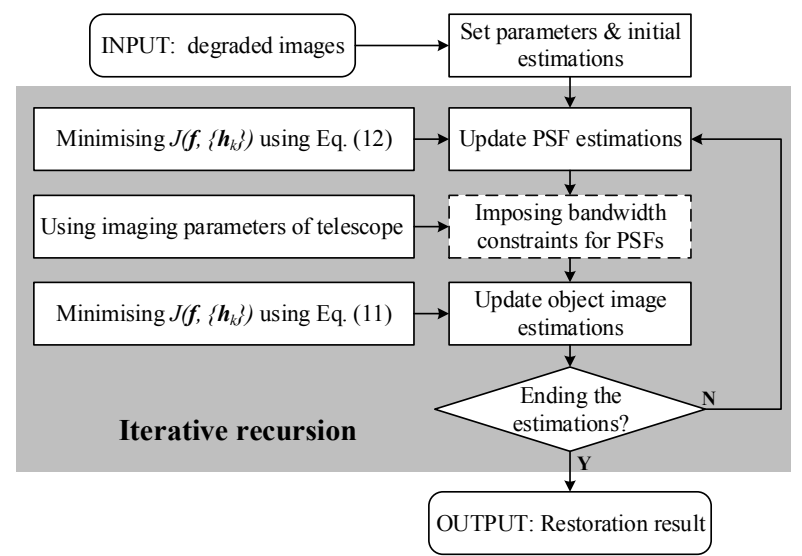

Fig. 1 Flowchart of MFRAM

Mixed noise model based MFBD: In order to reserve more details, the current ground-based telescopes usually grab multi-frame short-exposure images and use them to restore the object image. The process of the blurring effects (caused by the atmospheric turbulence) degrading the observed images can be viewed as an independent random process. The effect of atmospheric turbulence on an image can be considered as a linear shift-invariant system. In addition, the observed images are mainly corrupted by Poisson distributed quantum noise and additive Gaussian noise. The Poisson noise is signal-dependent [1], whereas the Gaussian noise is signal-independent [4]. Although Schulz et al. proposed to include a mixed noise model earlier [6], their approach is limited because 1) it only works when the parameters of the image system are fully known and 2) actually, their probability model was derived from Poisson noise, different to our approach. The mathematical model of the atmospheric turbulence degraded images with mixed noise can be described as:

$$
\boldsymbol{g}=\overline{\mathrm{P}}(\boldsymbol{h} \otimes \boldsymbol{f})+\mathrm{N}\left(\mathbf{0}, \sigma^{2} \cdot \mathbf{1}\right),
$$

where $\boldsymbol{g}, \boldsymbol{h}$ and $\boldsymbol{f}$ denote the observed image, the PSF and the object image, respectively. The $\overline{\mathrm{P}}(\cdot)$ represents a Poisson distribution and the variance of the additive Gaussian noise is $\sigma^{2}$. Since the intensities of the observed images are significantly larger than the Gaussian noise in Eq. (1), $\overline{\mathrm{P}}(\boldsymbol{h} \otimes \boldsymbol{f})$ can be approximated to a Gauss process according to the central limit theory, as $\overline{\mathrm{P}}(\lambda) \approx \mathrm{N}(\lambda, \lambda)$, and Eq. (1) is simplified to

$$
\boldsymbol{g}+\sigma^{2} \cdot \mathbf{1}=\overline{\mathrm{P}}\left(\boldsymbol{h} \otimes \boldsymbol{f}+\sigma^{2} \cdot \mathbf{1}\right) .
$$

In Eq. (2), each pixel in the degraded image $\boldsymbol{g}$ is an independent Poisson random variable with a mean $\mu=h \otimes f+\sigma^{2}$. For multi-frame acquisitions, the $K$-frame observed images are mutually independent, and the joint probability density distribution are

$$
\begin{aligned}
& \mathrm{p}\left(\left\{\boldsymbol{g}_{k}\right\} \mid\left\{\boldsymbol{h}_{k}\right\}, \boldsymbol{f}\right)=\prod_{k=1}^{K} \mathrm{p}\left(\boldsymbol{g}_{k} \mid \boldsymbol{h}_{k}, \boldsymbol{f}\right) \\
& =\prod_{k=1}^{K} \prod_{x, y} \frac{\left[\left(h_{k} \otimes f\right)(x, y)+\sigma_{k}^{2}\right]^{g_{k}(x, y)+\sigma_{k}^{2}} \exp \left[-\left(h_{k} \otimes f\right)(x, y)+\sigma_{k}^{2}\right]}{\left(g_{k}(x, y)+\sigma_{k}^{2}\right) !}
\end{aligned}
$$

The main task of the blind restoration is to estimate the object image $\boldsymbol{f}$ and PSFs $\left\{\boldsymbol{h}_{k}\right\}_{k=1}^{K}$ from the observed image $\left\{\boldsymbol{g}_{k}\right\}_{k=1}^{K}$ simultaneously. This involves solving the $(K+1)$ unknown parameters using only $K$ equations; this is a typical ill-posed inverse problem. According to the Bayes' law, the posterior probability density function (PDF) of the object image $f$ and the PSFs $\left\{\boldsymbol{h}_{k}\right\}_{k=1}^{K}$ on the observed images $\left\{\boldsymbol{g}_{k}\right\}_{k=1}^{K}$ is

$$
\mathrm{p}\left(\boldsymbol{f},\left\{\boldsymbol{h}_{k}\right\} \mid\left\{\boldsymbol{g}_{k}\right\}\right)=\frac{\mathrm{p}\left(\left\{\boldsymbol{g}_{k}\right\} \mid f,\left\{\boldsymbol{h}_{k}\right\}\right) \mathrm{p}\left(\boldsymbol{f},\left\{\boldsymbol{h}_{k}\right\}\right)}{\mathrm{p}\left(\left\{\boldsymbol{g}_{k}\right\}\right)} .
$$

The estimations of $\boldsymbol{f}$ and $\left\{\boldsymbol{h}_{k}\right\}_{k=1}^{K}$ are equivalent to finding the minimum of the negative logarithm likelihood of PDF, i.e.:

$$
\begin{aligned}
& \hat{\boldsymbol{f}},\left\{\hat{\boldsymbol{h}}_{k}\right\}=\underset{\boldsymbol{f},\left\{\boldsymbol{h}_{k}\right\}}{\arg \min }\left\{-\ln \left[\mathrm{p}\left(\boldsymbol{f},\left\{\boldsymbol{h}_{k}\right\} \mid\left\{\boldsymbol{g}_{k}\right\}\right)\right]\right\} \\
& =\underset{\boldsymbol{f},\left\{\boldsymbol{h}_{k}\right\}}{\arg \min }\left\{-\ln \left[\mathrm{p}\left(\left\{\boldsymbol{g}_{k}\right\} \mid \boldsymbol{f},\left\{\boldsymbol{h}_{k}\right\}\right)\right]-\ln [\mathrm{p}(\boldsymbol{f})]-\ln \left[\mathrm{p}\left(\left\{\boldsymbol{h}_{k}\right\}\right)\right]+\ln \left[\mathrm{p}\left(\left\{\boldsymbol{g}_{k}\right\}\right)\right]\right\}
\end{aligned}
$$

In Eq. (5), it is assumed that the object image $\boldsymbol{f}$ is independent to the PSFs $\left\{\boldsymbol{h}_{k}\right\}_{k=1}^{K}$, and $\mathrm{p}(\boldsymbol{f})$ and $\mathrm{p}\left(\left\{\boldsymbol{h}_{k}\right\}\right)$ are the a priori distributions of $\boldsymbol{f}$ and $\left\{\boldsymbol{h}_{k}\right\}$, respectively. $\boldsymbol{f}$ and the PSFs can be considered as the Gibbs random fields [7]. Their PDFs can be expressed as:

$$
\mathrm{p}(\boldsymbol{f})=e^{-\lambda J_{1}(\boldsymbol{f})} / C_{1}, \mathrm{p}\left(\left\{\boldsymbol{h}_{k}\right\}\right)=e^{-\beta J_{2}\left(\left\{\boldsymbol{h}_{k}\right\}\right)} / C_{2},
$$

where, $J_{1}(\boldsymbol{f})$ and $J_{2}\left(\left\{\boldsymbol{h}_{k}\right\}\right)$ are the energy functions, and $C_{1}$ and $C_{2}$ are constants. Combining Eqs (6) and (3), Eq. (5) is simplified as:

$$
\begin{aligned}
& \hat{\boldsymbol{f}},\left\{\hat{\boldsymbol{h}}_{k}\right\}=\underset{\boldsymbol{f},\left\{\boldsymbol{h}_{k}\right\}}{\arg \min }\left\{J\left(\boldsymbol{f},\left\{\boldsymbol{h}_{k}\right\}\right)\right\} \\
& =\underset{\boldsymbol{f}\left\{\boldsymbol{h}_{k}\right\}}{\arg \min }\left\{J_{0}\left(\boldsymbol{f},\left\{\boldsymbol{h}_{k}\right\}\right)+\lambda J_{1}(\boldsymbol{f})+\beta J_{2}\left(\left\{\boldsymbol{h}_{k}\right\}\right)\right\},
\end{aligned}
$$

where

$$
J_{0}\left(\boldsymbol{f},\left\{\boldsymbol{h}_{k}\right\}\right)=-\ln \left[\mathrm{p}\left(\left\{\boldsymbol{g}_{k}\right\} \mid \boldsymbol{f},\left\{\boldsymbol{h}_{k}\right\}\right)\right] .
$$

In Eq. (7), $J\left(\boldsymbol{f},\left\{\boldsymbol{h}_{k}\right\}\right)$ is the cost function of MFRAM. $J_{0}\left(\boldsymbol{f},\left\{\boldsymbol{h}_{k}\right\}\right)$ is the fidelity. $J_{1}(\boldsymbol{f})$ and $J_{2}\left(\left\{\boldsymbol{h}_{k}\right\}\right)$ are used for regularization. $\lambda$ and $\beta$ are non-zero constants. The Tikhonov regulation and the total variation (TV) regulation are applied to PSFs and $\boldsymbol{f}$ respectively to obtain:

$$
J_{1}(\boldsymbol{f})=\left\|\left.\nabla \boldsymbol{f}\left|\|_{1}, J_{2}\left(\left\{\boldsymbol{h}_{k}\right\}\right)=\sum_{k=1}^{K} \sum_{x, y}\right| h_{k}(x, y)\right|^{2} .\right.
$$

In this letter, an iterative recursive method is used to optimise $J\left(\boldsymbol{f},\left\{\boldsymbol{h}_{k}\right\}\right)$. The first order partial derivative of $J\left(\boldsymbol{f},\left\{\boldsymbol{h}_{k}\right\}\right)$ is derived from Eq. (7):

$$
\frac{\partial J\left(\boldsymbol{f},\left\{\boldsymbol{h}_{k}\right\}\right)}{\partial \boldsymbol{f}}=\sum_{k=1}^{K}\left[\left(1-\frac{\boldsymbol{g}_{k}+\sigma_{k}^{2}}{\boldsymbol{h}_{k} \otimes \boldsymbol{f}+\sigma_{k}^{2}}\right) \otimes \boldsymbol{h}_{k}^{*}\right]+\lambda \nabla J_{1}(\boldsymbol{f}),
$$

where $\boldsymbol{h}_{k}^{*}(x, y)=\boldsymbol{h}_{k}(-x,-y)$. The optimised condition can be found when $\partial J / \partial \boldsymbol{f}=0$, and we obtain the recursive form:

$$
\boldsymbol{f}^{(n+1)}=\frac{\boldsymbol{f}^{(n)}}{K+\lambda \nabla J_{1}\left(\boldsymbol{f}^{(n)}\right)} \sum_{k=1}^{K}\left(\boldsymbol{h}_{k}^{(n)^{*}} \otimes \frac{\boldsymbol{g}_{k}+\sigma_{k}^{2}}{\boldsymbol{h}_{k} \otimes \boldsymbol{f}+\sigma_{k}^{2}}\right) .
$$

A similar form can be obtained for the PSFs $\left\{\boldsymbol{h}_{k}\right\}_{k=1}^{K}$ :

$$
\boldsymbol{h}_{k}^{(n+1)}=\frac{\boldsymbol{h}_{k}^{(n)}}{1+\beta \nabla J_{2}\left(\boldsymbol{h}_{k}^{(n)}\right)} \cdot\left(\boldsymbol{f}^{(n)^{*}} \otimes \frac{\boldsymbol{g}_{k}+\sigma_{k}^{2}}{\boldsymbol{h}_{k} \otimes \boldsymbol{f}+\sigma_{k}^{2}}\right) .
$$


With an appropriate initial value, $J\left(\boldsymbol{f},\left\{\boldsymbol{h}_{k}\right\}\right)$ can be solved following the iterative recursion steps of Eqs (11) and (12). It is worth noting that, $J_{1}(\boldsymbol{f})$ and $J_{2}\left(\left\{\boldsymbol{h}_{k}\right\}\right)$ are constants if no regularization constraint is applied to $\boldsymbol{f}$ and $\left\{\boldsymbol{h}_{k}\right\}$, and $\sigma_{k}^{2}=0$ if we only consider Poisson noise. In this case, Eqs (11) and (12) are degenerated to the classic RichardsonLucy iterative blind deconvolution (RLIBD) algorithm. In fact, Eqs (11) and (12) are the expansion forms of RLIBD. In addition, the object image and the PSFs are physically constrained during the iterative procedure, that is, (a) the object image and the PSFs are non-negative; (b) the energy between observed image and object image remains the same; (c) the PSFs are bandwidth-limited functions due to the diffraction limits of the optical system. Fig. 1 shows the flowchart of MFRAM. Note that the imaging parameters of the ground-based telescopes should be known beforehand in order to enforce additional bandwidth restrictions to the PSFs. However, in some situations, the imaging parameters cannot be obtained, and therefore we cannot add bandwidth constraints to the PSFs in the deconvolution process, but our method still works efficiently although causing minimal degradation.

Experimental Results and Analysis: The image of the marine satellite OCNR5 was selected to validate the effectiveness of MFRAM. This image is frequently used for blind restoration of atmospheric turbulence degraded images. We also compared MFRAM with RLSATV [2] and OBD algorithms [4]. RLSATV and OBD are derived assuming that the noise is Poisson noise and Gaussian noise, respectively. We measured the image quality through both visual inspection and quantitative analysis. The quantitative measurements include the mean square error (MSE) and the peak signal-to-noise ratio (PSNR). The size of the original satellite OCNR5 is $256 \times 256$, as shown in Fig. 2 (a). The Zernike polynomials as well as appropriate Poisson noise and Gaussian noise were included to the images to simulate a series of atmospheric turbulence blurred images. A synthetic PSF and a degraded image are shown in Figs 2 (b) and 2 (c), respectively.
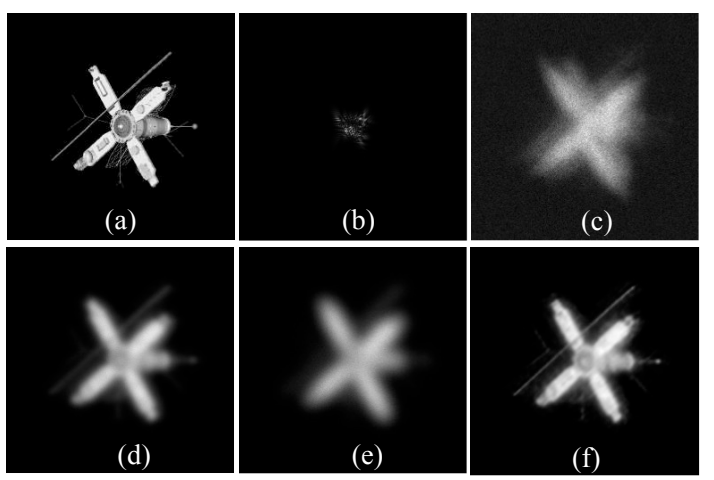

Fig. 2 Comparison of restoration results on Synthetic turbulence degraded images (a) Original image (b) Synthetic turbulence PSF (c) Synthetic turbulence-degraded image (d) RLSATV-restored image (e) $O B D$ - restored image (f) MFRAM -restored image

In the blind restoration experiment, 10 turbulence degraded images were selected as inputs. The regularization parameters: $\lambda=0.002$ $\beta=0.001$. The variance of Gaussian noise were calculated in the background area of the degraded images. We applied additional bandwidth constraints to the PSFs in the iterative process and compared the results of MFRAM with those obtained by RLSATV and OBD. The initial estimation of the object image is set as the average of the 10 input images. The initial conditions of the PSFs are set as the normalized constant matrix. The resultant object images are shown in Figs 2 (d), (e) and (f). The MSE and PSNR of the object image during the iterative process are illustrated in Figs 3 (a) and (b). Table 1 summarises the comparisons (in MSE and PSNR), and MFRAM shows the best results.

Figs 2 (d), (e) and (f) are the object images obtained by RLSATV, OBD and MFRAM, respectively. Through visual inspection, MFRAM can reduce the noise and distribute the image brightness more evenly, and it shows more details. From Fig. 2 and Table 1, MFRAM shows much better noise-removal and image quality than RLSATV and OBD. Fig. 3 shows that an excellent restoration result is obtained by MFRAM after only 150 iterations. It is faster than RLSATV, much faster than OBD.

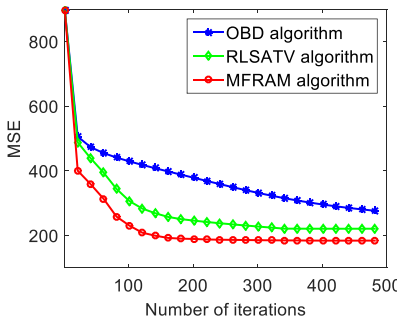

(a)

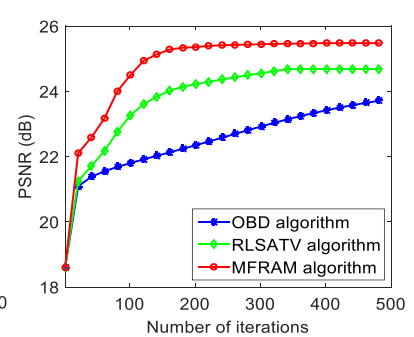

(b)
Fig. 3 MSE and PSNR for the estimated object image with respect to the number of iterations

Table 1: Comparisons of the blind deconvolution results

\begin{tabular}{lccc}
\hline & RLSATV & OBD & MFRAM \\
\hline MSE & 221.0 & 276.6 & 184.2 \\
PSNR(dB) & 24.68 & 23.71 & 25.48 \\
\hline
\end{tabular}

Conclusions: In this letter, we proposed a novel algorithm named MFRAM to effectively restore the true object image from a sequence of turbulence-degraded images. Using the Bayesian framework, the general cost function of the multi-frame image blind deconvolution with a mixed noise model was derived. An iterative recursion method was adopted to solve the cost function. Three limited bandwidth constraints of PSFs were included to the recursion process to avoid converging to local minima. We compared the results of MFRAM to well-known RLSATV and OBD. The results show that, MFRAM can achieve a faster convergence speed and preserve more image details.

Acknowledgments: This work was supported by National Natural Science Foundation of China (61601488), Hunan Provincial Natural Science Foundation of China (2016JJ3024), Science Research Plan Project of National University of Defense Technology (ZK16-03-08) and The Open Foundation of State Key Laboratory of Astronautic Dynamics (2016ADL-DW0301).

Afeng Yang (College of Basic Education, National University of Defense Technology, Changsha, China; Centre for Biophotonics, University of Strathclyde, Glasgow, United Kingdom)

Email: yangafeng@nudt.edu.cn

Xue Jiang (Department of Naval Architecture, Ocean and Marine Engineering, University of Strathclyde, Glasgow, United Kingdom)

David Day-Uei Li (Centre for Biophotonics, University of Strathclyde, Glasgow, United Kingdom)

\section{References}

1. Matson, C. L., Borelli, K., Jefferies, S. M., et al.: 'Fast and optimal multiframe blind deconvolution algorithm for high-resolution groundbased imaging of space objects', Applied Optics, 2009, 48, (1), pp. A75A92

2. Yan, L., Fang, H. and Zhong, S.: 'Blind image deconvolution with spatially adaptive total variation regularization', Optics Letters, 2012, 37, (14), pp. 2778-2780

3. Vorontsov, S. V. and Jefferies, S. M.: 'A new approach to blind deconvolution of astronomical images', Inverse Problems, 2017, 33, (5), 055004 (21pp)

4. Hirsch, M., Harmeling, S., Sra, S., et al.: 'Online multi-frame blind deconvolution with super-resolution and saturation correction', Astronomy \& Astrophysics, 2011, 531, A9 (11pp)

5. Li, D., Sun, C., Yang, J., et al.: 'Robust multi-frame adaptive optics image restoration algorithm using maximum likelihood estimation with poisson statistics', Sensors, 2017, 17, (4), 785 (19pp)

6. Schulz, T. J., Stribling, B. E. and Miller J. J.: 'Multiframe blind deconvolution with real data: imagery of the Hubble Space Telescope', Opt Express, 1997, 1, (11) pp. 355-362

7. Bertero, M., Boccacci, P., Desiderà, G., et al.: 'Image deblurring with Poisson data from cells to galaxies', Inverse Problems, 2009, 25, 123006. 